\title{
Palliative Care, Suffering, Death Trajectory: A View of End-of-Life Care (EOL) Related Issues in Sub-Saharan Africa (SSA)
}

\author{
Albert M. E. Coleman'1,2 \\ ${ }^{1}$ Southern Health NHS Foundation Trust, Winchester Older Person Mental Health Community Services, Winchester, UK \\ ${ }^{2}$ Centre for Medical Law, Bioethics and Patient Advocacy, Sekondi, Ghana \\ Email: albert.coleman@gmail.com
}

How to cite this paper: Coleman, A.M.E. (2018) Palliative Care, Suffering, Death Trajectory: A View of End-of-Life Care (EOL) Related Issues in Sub-Saharan Africa (SSA). International Journal of Clinical Medicine, 9, 175-181.

https://doi.org/10.4236/ijcm.2018.93015

Received: December 18, 2017

Accepted: March 16, 2018

Published: March 19, 2018

Copyright $\odot 2018$ by author and Scientific Research Publishing Inc. This work is licensed under the Creative Commons Attribution International License (CC BY 4.0).

http://creativecommons.org/licenses/by/4.0/

\begin{abstract}
Palliative care in the sub-Saharan Africa (SSA) region despite some progress made since the first hospice was opened in Zimbabwe in 1979, still lags far behind that of countries with developed economies, and relatively suffers from not being wholly included into mainstream public health service delivery in SSA. The situation is made worse due to relatively poor and pervasive sociopolitico-economic factors and the challenge of the changing and increasing non-communicable disease epidemiology in SSA countries. This situation results in a tension between scarce resources and service needs/provision which prevails in a good number of SSA countries. In large part the situation where palliative care, end of life and the death trajectory converge in SSA countries currently portrays one of scarcity of resources and suffering for those ill SSA patients who need the services. This article is an overview of the current situation as pertains to palliative care services in the SSA region and some of the factors that contribute to or perpetuate the current state of palliative care delivery in SSA countries.
\end{abstract}

\section{Keywords}

End-of-Life, Palliative Care, Sub-Saharan Africa, Socio-Economic Poverty, Suffering

\section{Introduction}

End-of-life (EOL) care issues in lower and middle income countries (LMIC) especially in Sub-Saharan Africa (SSA), can be problematic compared to EOL management standards in developed economy countries [1] [2] [3]. This situation is influenced by socio-economic problems, a relatively high infectious dis- 
ease burden and an increasing chronic disease burden [4], and hence different national healthcare priorities and policies in LMIC, especially SSA countries. This state of affairs potentially places EOL care in the LMIC in a lower policy priority compared to other national healthcare sector policies. Except for cases of terminal diseases in the young and working groups EOL matters most often are issues linked to the elderly population strata in countries with developed economies, where one meets a lot of chronic and co-morbid chronic illnesses and or a relatively higher incidence of cancers [5] [6]. This is not necessarily the case in SSA countries.

\section{Contributory Factors to the Current EOL Situation in the SSA Region}

\subsection{Mortality and Morbidity Factors}

Mortality and morbidity indices for countries tend in general to be unfavourable in LMIC especially in SSA countries and are not necessarily predominant in the older age strata compared to those of developed economy countries [4] [6] [7]. This is due to some factors that have been previously mentioned as contributing to poor EOL care in those countries. In addition to those factors already mentioned, other contributory considerations include health behaviour and illness locus of control factors and human and material resources etc.

\subsection{Traditional Beliefs, SSA Communitarianism and Locus of Illness Concept}

Especially for SSA countries an important consideration impacting on EOL care matters and hence palliative care service delivery is the traditional/cultural beliefs and practices (CBP) about illness and death and EOL [8] [9] [10], relatively complex factors compared to health and illness behaviour in most developed economy countries. Generally CBP in most SSA settings around issues of death/EOL or death trajectory are centred around the concept of community [8] [9], a belief in spiritism and or witchcraft or evil spirits [9] [10], and the concept in some instances of the departing individual being a bridge between the departed (dead) and the living community [8] [9] (more on that in a later section). SSA CBP is largely based on the concept of communitarianism, where individual patient decisions in a good number of instances take on a "relational" aspect, involving family [11] [12] [13]. This relational aspect may even be extended to an inter-relational one, whereby the family members of the patient on the death pathway albeit deemed mentally to have capacity to make decisions, may choose to overstep the patient and engage the health care professionals (HCP) as to patient EOL related matters. A situation that may be considered privy to the patient in developed economy country settings. This may create a clash between the ethical principle of respect for the patient's autonomy, communitarian practices and the doctor-patient relationship and confidentiality, raising issues of conflict for the treating HCP. 
Another dimension of this is the patient/community view/understanding of health behaviour and inherently illness locus of control [10] [14]. This is important as illness locus of control and illness behaviour go hand in hand in most cases to determine how patients look on and respond to their illness, and hence EOL management in cases of terminal illness. Hence in a good number of SSA countries belief in sprits and witches as illness causative factors [8] [9] [10], may determine where terminally ill persons go to seek help/treatment. Terminally ill patients at EOL may then end up with the witch doctor without access to appropriate in most palliative care instances.

\section{Current Extent of Palliative Care Practice in SSA Countries}

Palliative care at EOL in SSA countries can be considered a mixed picture, with most SSA countries without any service, few in various stages of development (mostly on paper plans, or with a few declared professional interest) [1] [2] [3]. Since the first recorded palliative care service was set up in Zimbabwe in 1979 [15] the Eastern SSA Sub-Region (Tanzania, Uganda, Kenya) as well as the Republic of South Africa (RSA) have forged a path towards setting up Services, other services are being set up in other parts of SSA [16] [17]; albeit most not yet up to standards of those in developed economy nations. In a good number of these settings, palliative care is actually being undertaken in the community by family members given some basic training by non-governmental organisations, and not as a mainstream public health initiative [18]. In short the situation in SSA countries is one of relative scarcity of palliative care services and resources'.

\section{SSA Healthcare Delivery, CBP and the Relation to EOL}

Traditional views and cultural systems in SSA countries with the pervasive belief in spiritism, gods and witchcraft and their role in illness [9] [10] impact on SSA citizenry views on the death trajectory, terminal illness and management of EOL. This could even be reflected in the practice habits of healthcare personnel (HCP) in such a setting, were he/she to belief in spirits, gods and witchcraft-which inevitably happens in some cases. In such a situation selling conventional allopathic driven palliative care tenets to EOL patients in such settings can be anything but challenging. Attitudes of fatalism and determinism may intervene or come into play, creating additional levels of complexity.

There are many anecdotal stories from SSA countries healthcare settings (I happen to have first-hand knowledge in my native African country). There have been several incidents where on a patient's own wishes, but many a time on the urges of relatives, terminally ill patient's in hospital ask to be discharged primarily because they are "going to seek alternate help", most times with premature adverse outcomes. Even in current times the influence of tradition/culture is still real. Conversely, it can sometimes be the HCP managing terminally ill patients who because of their own entrenched cultural beliefs will ask families of patients on the death pathway to "take the patient home". Decoded this interprets as 
"there is not much the HC team can offer from the point of view of allopathic medicine, so take the patient home and try something else (traditional medicine, witch doctors etc)". This clearly is not the attitude in most developed economy countries where when terminal patients are being discharged home appropriate transitional care arrangements will be put in place, to ensure evidence based appropriate community palliative care [19].

\section{Changing Demographics and EOL in SSA Settings}

SSA demographic make-up is slowly but gradually changing to reflect a relatively small but ever growing elderly population compared to the previous years' "pyramid" character [20]. I doubt though that most SSA health systems are ready for a gradually growing older generation. As mentioned earlier along with ageing comes a relatively higher prevalence of chronic, often debilitating chronic diseases, relatively high rates of cancer [5] [6] [7] and by extension increasing demand for specialised EOL care linked to disease burden of aging. Combining this challenge of slow but emerging demographic change with the traditional/cultural attitude of SSA countries towards the death pathway and EOL management shows the magnitude of the challenge facing these countries, where palliative services implementation is concerned.

\section{Problem of Socio-Economic Poverty and Resource Scarcity on EOL in SSA Countries}

Despite some progress, most SSA countries continue to face socio-economic problems as well as relatively poor governance, which inadvertently impacts on resources (human, financial and material) [4] meant to shore up and maintain appropriate and credible healthcare delivery (including EOL and palliative care services). This pervasive situation contributes to and compound problems leading to inequalities in health [4] and healthcare delivery and services, including EOL care.

\section{Ethical Dimension of Lack of or Relatively Poor Palliative Care/EOL Services for SSA Citizenry}

Compared to the citizenry of developed economy countries the reality of day to day living for most SSA citizenry is relatively tough though not necessarily doom and gloom. The several challenges to human personhood in these settings in large part is that of a relatively large vulnerable population ready to fall "through the web of vulnerability", paraphrasing from Tronto [21], where there is no "safety net" to break the fall. In such a situation existential problems pose a threat to human dignity. Extended to the death trajectory in situations of palliative care/EOL in SSA settings, EOL can be difficult with the suffering index relatively pronounced compared to those in the developed countries. Such a situation poses a heightened risk for SSA HCP towards developing moral distress and compassion fatigue [22], in carrying out their day to day task. The impact of socio-economic and caring pressures combined with the collapse of the often al- 
luded to SSA community support network [23] means that the growing needs of carers involved in SSA palliative care delivery must also be taken into account.

\section{Discussion}

In ensuring appropriate and adequate palliative care services along the death trajectory for EOL patients in SSA, health policy makers, managers and carers are faced with hard choices against other coexistent population health needs [4]. In such a setting competing factors of the type of "needs versus resources" gives rise to "tensions" which call on HC managers and policy makers to make hard choices. The pressing needs of high infant/maternal mortality, human immune deficiency (HIV) high prevalence rates and emerging deadly zoonosis disease (example, Ebola) etc [6] [24] frequently leads to the situation where the development of appropriate but under-resourced palliative care services is deferred indefinitely for future consideration (hopefully), or be non-existent. In this scenario SSA healthcare systems may consider palliative care services to be an exotic speciality/service need more suited to the developed economy countries (at least for now). Unfortunately terminal illnesses, and consequently death are not a preserve only of countries with developed economies but an inevitable occurrence in all socio-economic systems. For all the reasons enumerated previously that stand in the way of SSA countries from providing evidence based EOL services, and hence effective palliative care services in these settings; most SSA citizens potentially face a bumpy death trajectory that can be characterised as one of suffering of patients and scarcity of resources.

\section{Conclusion}

Despite competing healthcare systems demands in SSA counties because of the "needs versus resource" tensions, health care policy makers/managers in SSA countries need to work on the issue of a dignified, death trajectory at EOL that is as free of pain and suffering as possible for its citizenry, whilst continuing to battle the ravages of preventable and chronic illnesses known to contribute to high mortality and morbidity. In so doing they should be careful not to be caught up in setting up EOL or palliative care services that will assume a "business role", as opposed to a value based palliative care system. In developing viable "development appropriate" palliative care services, SSA countries can seek assistance from organisations in developed economies with the appropriate experience and expertise in setting up appropriate cost saving palliative care systems, especially in the public health sector. For the citizens of SSA countries who are challenged day to day by existential difficulties the creation of a working, ably managed palliative care service may allow them find peace and dignity at EOL.

\section{Acknowledgements}

I thank S.C. for making time to proof read this article and for the helpful comments that she made towards improving the style and writing of this article. 


\section{Declaration of Interest}

None declared.

\section{Authorship}

The author single handily conceived the theme of the article and wrote the whole article.

\section{Ethical Approval}

Not applicable in this case.

\section{References}

[1] Clark, D. (2007-2008) End-Of-Life Care around the World: Achievements to Date and Challenges Remaining. Omega, 56, 101-110.

[2] Wright, M. and Clark, D. (2006) Hospice and Palliative Care Development in Africa: A Review of Development and Challenges. Oxford University Press, Oxford. https://doi.org/10.1093/acprof:oso/9780199206803.001.0001

[3] Murray, S.A., Grant, A. and Kendall, M. (2003) Dying from Cancer in Developed and Developing Countries Lessons from Two Qualitative Interview Studies of Patients and Carers. BMJ, 326, 1-5. https://doi.org/10.1136/bmj.326.7385.368

[4] Marmot, M. (2005) Social Determinants of Health Inequalities. Lancet, 365, 1099-104.

[5] Chrisstensen, K., Doblhammer, G., Rau, R. and Vaupel, J.W. (2009) Ageing Populations: The Challenges Ahead. Lancet, 374, 1196-1208. https://doi.org/10.1016/S0140-6736(09)61460-4

[6] Mathers, C.D. and Loncar, D. (2006) Projections of Global Mortality and Burden of Disease from 2002 to 2030. PLoS Medicine, 3, e442.

https://doi.org/10.1371/journal.pmed.0030442

[7] World Health Organization (WHO) (2009) Mortality and Burden of Disease States Estimates For Who Member States in 2004. WHO, Geneva.

[8] Gyeke, K. (1995) An Essay on African Philosophical Thought. The Akan Conceptual Scheme. Temple University Press, Philadelphia, PA.

[9] Tenkorang, E.Y., Gyimah, S.O., Maticka-Tyndale, E. and Adjei, J. (2001) Superstition, Witchcraft and HIV Prevention in Su-Sahara Africa: The Case of Ghana. Culture Health and Sexuality, 13, 1001-1014.

[10] Liddell, C., Barrett, L. and Bydawell, M. (2005) Indigenous Representation of Illness and AIDS in Sub-Saharan Africa. Social \& Medicine, 60, 691-700. https://doi.org/10.1016/j.socscimed.2004.06.020

[11] Mbiti, J.S. (1969) African Religions and Philosophy. East African Educational Publishers Ltd., Nairobi.

[12] Maina, W.M. (2008) African Communitarian Ethics in the Theological Work of Benezet Bujo. Pacifica, 21, 192-209. https://doi.org/10.1177/1030570X0802100205

[13] Behrens, K.G. (2013) Towards an Indigenous African Bioethics. $S A J B L$, 6, 32-35.

[14] Wallston, B.S. and Wallston, K.A. (1978) Locus of Control and Health: A Review of the Literature. Health Education Monographs, 6, 107-117.

[15] Stijernsward, J. and Clark, D. (2003) Palliative Care a Global Perspective. In: Hanks, 
G., Doyle, D. and Calman, C.N. Eds., Oxford Textbook of Palliative Medicine, 3rd Edition, Oxford University Press, Oxford.

[16] Grant, L., Downing, J., Namukwaya, E., Leng, M. and Murray, S. (2011) Palliative Care in Africa since 2005: Good Progress But Much Further to Go. British Medical Journal Supportive \& Palliative Care, 1, 118-122. https://doi.org/10.1136/bmjspcare-2011-000057

[17] Harding, R., Selman, L., Powell, R.A., Namisango, E., Downing, J., Merriman, A., et al. (2013) Research into Palliative Care in Sub-Saharan Africa. The Lancet Oncology, 14, e183-e188. https://doi.org/10.1016/S1470-2045(12)70396-0

[18] Streid, J., Harding, R., Agupi, G., Dinat, N., Downing, J., Gwyther, L., et al. (2014) Stressors and Resources of Caregivers of Patients with Incurable Progressive Illness in Sub-Saharan Africa. Qualitative Health Research, 24, 317-328. https://doi.org/10.1177/1049732314523682

[19] Reunke, S., Nguyen, O.K., Shoeb, M.H., Magan, Y. and Wachter, R.M. (2013) Hospital-Initiated Transitional Care Intervention as a Patient Safety Strategy. Annals of Internal Medicine, 158, 433-441.

[20] Cohen, B. and Menken, J. (2006) Ageing in Sub-Saharan Africa: Recommendations for Further Research. National Academies Press, Washington, DC.

[21] Tronto, J. (2001) An Ethic of Care. Ethics in Community-Based Elder Care. Springer Publishing Company, New York, 60-68.

[22] Harrowing, J.N. and Mill, J. (2010) Moral Distress among Ugandan Nurses Providing HIV Care: A Critical Ethnography. International Journal of Nursing Studies, 47, 723-731. https://doi.org/10.1016/j.ijnurstu.2009.11.010

[23] Gysels, M., Pell, C. and Pool, R. (2011) End of Life Care in Sub-Saharan Africa: A Systematic Review of the Qualitative Literature. BMC Palliative Care, 10, 6. https://doi.org/10.1186/1472-684X-10-6

[24] Boutayeb, A. (2006) The Double Burden of Communicable and Non-Communicable Diseases in Developing Countries. Transactions of the Royal Society of Tropical Medicine and Hygiene, 100, 191-199. https://doi.org/10.1016/j.trstmh.2005.07.021 